\title{
Reachability Analysis of Time Basic Petri Nets: a Time Coverage Approach
}

\author{
Carlo Bellettini, Lorenzo Capra \\ Department of Informatics and Communication \\ Università degli Studi di Milano, Italy \\ Email: \{bellettini, capra\}@dico.unimi.it
}

\begin{abstract}
We introduce a technique for reachability analysis of Time-Basic (TB) Petri nets, a powerful formalism for realtime systems where time constraints are expressed as intervals, representing possible transition firing times, whose bounds are functions of marking's time description. The technique consists of building a symbolic reachability graph relying on a sort of time coverage, and overcomes the limitations of the only available analyzer for TB nets, based in turn on a time-bounded inspection of a (possibly infinite) reachability-tree. The graph construction algorithm has been automated by a tool-set, briefly described in the paper together with its main functionality and analysis capability. A running example is used throughout the paper to sketch the symbolic graph construction. A use case describing a small real system - that the running example is an excerpt from - has been employed to benchmark the technique and the tool-set. The main outcome of this test are also presented in the paper. Ongoing work, in the perspective of integrating with a model-checking engine, is shortly discussed.
\end{abstract}

\section{INTRODUCTION}

Time-Basic (TB) Petri nets [1] belong to the category of nets in which system time constraints are expressed as numerical intervals associated to each transition, representing possible firing instants, computed since transition's enabling time. Tokens atomically produced by the firing of a transition are thereby associated to time-stamps with values ranging over a determined set. With respect to the well-known representative of this category, i.e., Time Petri nets [2], interval bounds in TB nets are linear functions of timestamps in the enabling marking, rather than simply numerical constants. TB nets thus represent a much more expressive formal model for real-time systems. The reachability analysis of TB nets is still recognized as an open problem ([3]). Available analysis techniques and tools (e.g., [3], [4]) are based on inspecting a finite portion of the potentially infinite reachability-tree generated by a TB net. But for particular cases, only time-bounded properties can be inferred from TB net's state-space exploration by using this kind of analyzers. The technique described in this paper tries to overcome this major limitation. It relies on a symbolic reachability graph algorithm, which is in turn based on a relative notion of time and a procedure verifying inclusion between symbolic states. A particular state normalization, able to recognize and eliminate timestamp symbols actually not influencing the model evolution, permits in many cases building a sort of time coverage finite graph. The symbolic graph construction has been automated by a tool-set written in Java. The output is a structure enriched with information on edges which might be exploited during property evaluation. The tool-set currently includes a module for the automatic verification of properties expressed as conditions on markings. As use case we'll use the gas burner example, that is widely used in literature as a representative of a small real system. A complete and formal description can be found in [5], and the corresponding TB net model was introduced in [6]. An excerpt will be used as running example to explain in a rather informal way the essential points of symbolic graph construction. Only some relevant new core definitions are formally given.

\section{TBNETS}

Time Basic nets are Petri nets where each token is associated with a time-stamp representing the instant at which it has been created. In this paper we assume that the domain of timestamps is $\mathbb{R}^{+}$. Each transition $t$ is associated with a time function $f_{t}$ which maps a tuple en of time-stamps, one for each place in - $t$ (the pre-set of $t$ ), to a (possibly empty) set of $\mathbb{R}^{+}$values. A marking $m$ maps each place $p$ to a multi-set in $\mathbb{R}^{+}$. A tuple $e n$ is said to be an enabling tuple for $t$ in $m$ if $m$ contains $e n$ and $f_{t}(e n) \neq \emptyset$. The set $f_{t}(e n)$ represents the possible firing times of enabling tuple $e n$. The firing of $(e n, t)$ makes $e n$ be withdrawn from $\bullet t$, and a new timestamp arbitrarily chosen among the values in $f_{t}(e n)$ be created in all places in $t^{\bullet}$ (the post-set of $t$ ).

Hereafter a time function $f_{t}$ is defined by a pair of linear functions $\left[l b_{t}, u b_{t}\right]$, denoting interval bounds. $l b_{t}, u b_{t}$ are in turn formally expressed in terms of (a non empty set of) names of places in $t$. Time-functions are monotonic, i.e., the set of time-stamps associated with a tuple en cannot contain a timestamp less than the maximum time-stamp associated with a token in en, denoted enab. We will keep such assumption implicit in the formal notation for time-functions.

Consider the excerpt from the use case, depicted in Fig. 1 It relates to the Ignite Phase, just after the ignition transformer has been started and the gas valve has been opened. In this phase the controller must check if the flame has been lighted within a specific deadline, otherwise a recovery procedure that brings the system to Idle has to be activated. The flame turns on if there are Ignition and Gas (transition FlameLigthOn), but it can turn off if no gas is supplied (transition FlameLigthOff) or due to a fault, e.g. some wind (transition FlameLigthOff2). The time function associated with transition FlameOn (representing the system passing to burnstate after recognizing 

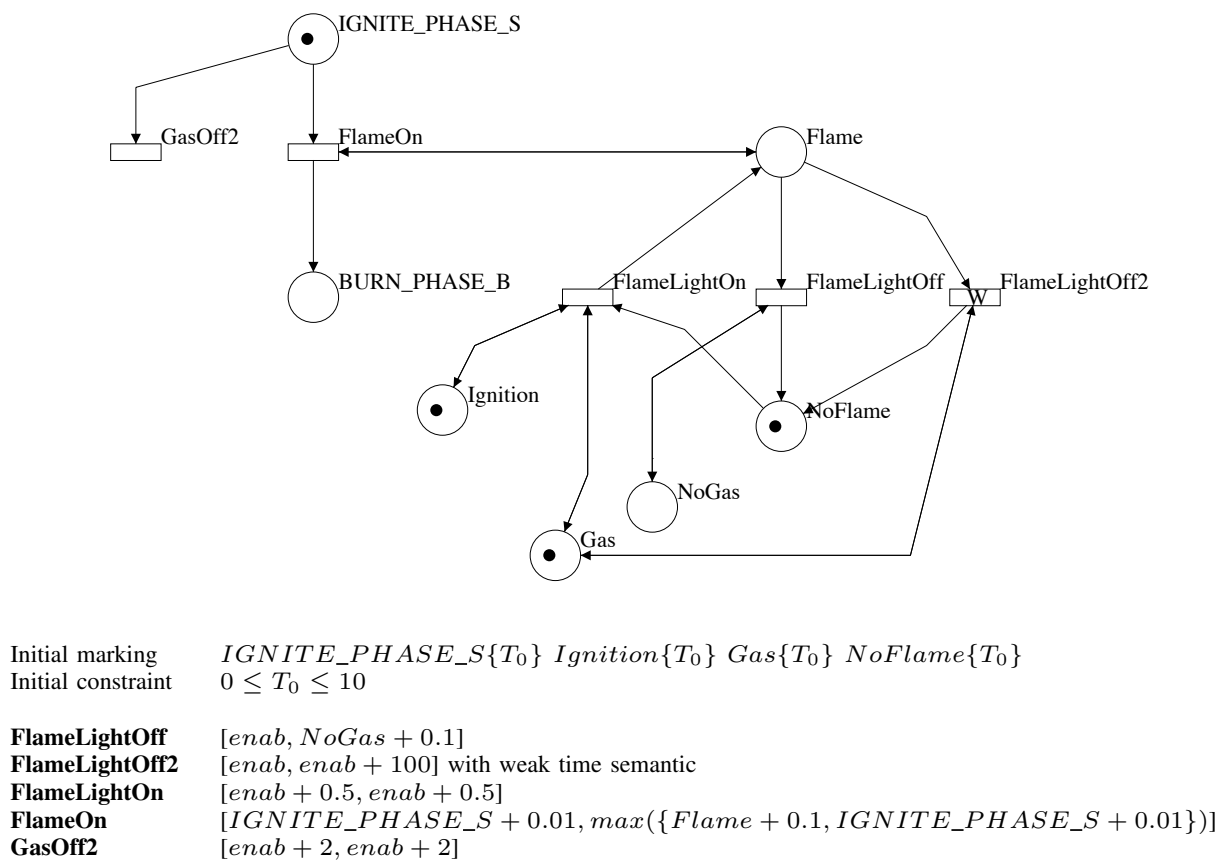

Fig. 1. running example

that the flame has turned on) can be interpreted as follows: FlameOn cannot fire before 0.01 time units elapse since the appearance of a token in place IGNITE_PHASE_S (the minimum permanence time in ignitestate) and implicitly not before the timestamp in place Flame. The firing time cannot exceed the maximum between the timestamp of the token in place IGNITE_PHASE_S plus 0.01 time units and the time-stamp of the token in place Flame plus 0.1 (i.e., the system recognizes the presence of a flame within this 0.1 units). Noticeably, this is an example of constraint that cannot be directly expressed using Time Petri Nets formalism ([2]).

The set of time-stamps associated with a tuple by $f_{t}$ can be interpreted in at least two different ways, leading to different time semantics for each transition. A first interpretation states that an enabled transition $t$ can fire at a value included in the set of possible firing times. Transitions with such semantics are referred to as weak transitions. A second interpretation states that an enabled transition $t$ must fire at a value included in the set of possible firing times unless it is disabled by the firing of any conflicting transition at a time no greater than the greatest firing time of $t$. Transitions with such semantics are referred to as strong transitions. Concerning the net in Fig. 1 . the only weak transition is FlameLightOff2. This permits us to express the possibility that an event occurs within a given time interval. In the case of Time Petri Nets the only possible semantics is strong.

In order to meet an intuitive notion of time, TB net firing sequences are restricted to the set of firing sequences whose firing times are monotonically non decreasing with respect to the firing occurrences. However, the time of a firing may be equal to the enabling time of the tuple that belongs to the firing. Intuitively this means that an effect (the firing) can occur with no delay after the cause (that enables it) is fulfilled. Therefore, it is possible to have sequences of firings where the time does not change. In practice, it is useful to restrict the attention to a subclass of TB nets, such that there exist no infinitely long firing sequences which take a finite amount of time (non Zenonicity).

\section{TIME COVERAGE REACHABILITY ANALYSIS}

The analysis technique presented in this paper extends the capability of the existing analyzer for TB nets [7], which uniquely permits the verification of bounded invariance and response properties, through the inspection of a time-bounded symbolic reachability tree generated from a TB net.

The new technique aims at building a finite graph instead of an infinite tree for a wide category of TB nets. A combination of three complementary ideas is exploited. First, symbolic states are compared to check subset relationships. For that purpose, using a consolidated approach, timestamp symbols no more occurring on the marking description are eliminated from the linear constraint associated to a symbolic state, independently of how it has been reached. Identifying subset relations between generated symbolic states (markings plus constraints), is necessary for recognizing cyclic paths, but it is not enough in many situations. As time progresses, periodic occurrences of equivalent conditions may be unrecognizable simply due to their different offsets with respect to system's time zero. This observation leads us dealing with the second aspect. In the very common case a TB model contains no reference to absolute times (i.e., not as offset respect to enabling timestamps) in transition time functions, it is possible to remove any references to the "absolute zero" from symbolic 
states. This permits a periodic equivalent behavior to be recognized. The cost is a lossy information about state displacement along absolute time. We'll discuss this aspects in section IV Let us only point out that this kind of information could be recovered, if necessary, in a second step by retracing only the path(s) leading to the state of interest, or (at least partially) by combining the information on edges. The third key feature of the technique is the introduction of the time anonymous (TA) concept. This relates to the fact that in a symbolic state there may exist tokens whose timestamp values can be forgotten, as not influencing the evolution of a model. Several heuristics have been implemented, based on a mix of structural and state-dependent patterns, each characterizing one such situation. This enhances the ability of merging states, and permits facing situations where the presence of dead tokens could reintroduce a sort of symbolic absolute zero, nullifying the achievements at the previous points. Again, the cost to pay is a minor loss of information, as discussed later. There is some resemblance with the approach used in the construction of (topological) coverage graphs: the missing information is the exact timestamp of tokens instead of their exact number. TA recognition might be also exploited to introduce a topological notion of coverage for TB nets (section VII).

\section{A. Graph construction}

In order to understand the rationale behind the symbolic reachability graph construction technique for TB nets, we shall use once again the running example in Fig. 11. Let us only introduce a few basic notions used in the sequel, referring to [8] (where the symbolic reachability tree for TB nets is defined) for a full formalization.

Let $T S=\left\{T_{i}\right\}, i \geq 0$, be the set of time-stamp symbols. A symbolic state $S$ is a pair $\langle M, C\rangle$, where $M$ (called marking) maps each place $p$ to a multi-set on $T S$, and $C$ is a (satisfiable) linear constraint defined on a subset of $T S$ symbols appearing in $M$. We are considering a normal form of $S$ : if $M$ contains $k$ different $T S$ symbols, they are $\left\{T_{0}, \ldots, T_{k-1}\right\}$, with the (implicit) assumption $\forall i: 0 \ldots k-2, C \Rightarrow T_{i} \leq T_{i+1}$. Unless otherwise specified, we shall refer to this form.

A mapping $e n_{s}:{ }^{\bullet} t \rightarrow T S$ is said a symbolic instance of $t$ (the notation $\left(e n_{s}, t\right)$ will be sometimes used). $e n_{s}$ will be formally denoted by a tuple of symbols. A symbolic evaluation of a linear function $g_{t}$ appearing in the formal definition of a time function, denoted $g_{t}\left(e n_{s}\right)$, is obtained by replacing each occurrence of $p \in{ }^{\bullet} t$ in the formal expression of $g_{t}$ with the associated symbol $\tau=e n_{s}(p)$.

According to a (monotonic) weak time semantics, a pair $\left(e n_{s}, t\right)$ is said a symbolic enabling in $S$ if $M$ contains $e n_{s}$ and $C^{\prime}: C \wedge l b_{t}\left(e n_{s}\right) \leq T_{k} \leq u b_{t}\left(e n_{s}\right) \wedge T_{k-1} \leq T_{k}$ is satisfiable. In other words, there exists at least one substitution $e n$ of numerical values for $e n_{s}$ that makes $C$ satisfiable and the set $f_{t}(e n)$ non empty.

The firing of symbolic enabling $\left(e n_{s}, t\right)$ produces the new symbolic state $S^{\prime}:\left\langle M^{\prime}, C^{\prime}\right\rangle$, where $M^{\prime}$ is obtained from $M$ by removing $e n_{s}$ and putting a new symbol $T_{k}$ in all places in $t^{\bullet}$. The state $S^{\prime}$ represents all the possible TB net ordinary markings reachable from any marking represented by $S$ by means of any firing correponding to $\left(e n_{s}, t\right)$.

\section{B. Time-coverage graph}

The time-coverage symbolic reachability graph generated by the running example, composed by 14 symbolic states, is presented in Fig. 211

The adopted notation for states is: a square for symbolic states, a double square for symbolic states containing some deadlocks. Concerning edges, the format of head and tail specifies the kind of relation between source and target.

The normal case, corresponding to a symbolic enabling, is black head and tail, e.g., from $S 0$ to $S 1$ : considering any marking represented by $S 0$ it is always possible to follow that edge and reach all the markings represented by $S 1$.

Let us consider the symbolic state $S 8$, formally described as follows:

$$
\begin{aligned}
\text { M8 }: & \text { Gas }\left\{T_{1}\right\} \text { IGNITE_PHASE_S } S\left\{T_{0}\right\} \\
& \text { Ignition }\{T A\} \text { NoFlame }\{T A\} \\
C 8: & T_{1} \geq T_{0}+1.5 \wedge T_{1} \leq T_{0}+1.8
\end{aligned}
$$

We can observe that, with respect to the original definition of symbolic state, a first extra time-stamp symbol is present, $T A$ (time anonymous). This new symbol can occur only on the marking. Postponing an intuitive explanation of when and how symbol $T A$ is introduced in a symbolic state representation, we can think of it as a token carrying on an unspecified timestamp, which has been shown unessential for the computation of transition firing times.

The "candidates" for symbolic enabling in $S 8$ are: $\left(\left\langle T_{0}\right\rangle\right.$, GasOff2) and $\left(\left\langle T A, T_{1}, T A\right\rangle\right.$, FlameLightOn $)$. Firing times are computed by (symbolically) evaluating transition time functions, as explained above. For GasOff2 the (only) inferred firing time is $\left\{T_{0}+2\right\}$. Time function evaluation is slightly different for FlameLightOn, due to the occurrence of $T A$ in the pre-set tuple: this symbol is simply erased during (symbolic) evaluation: enab $=\max \left(\left\{T A, T_{1}, T A\right\}\right) \equiv$ $\max \left(\left\{T_{1}\right\}\right)=T_{1}$. The inferred firing time in this case is $\left\{T_{1}+0.5\right\}$.

Since both transitions have a strong semantics, there are two additional constraints specifying that the firing time of one cannot be greater than the (maximum) firing time of the other ${ }^{2}$ They are $C_{\mathrm{GO} 2}: T_{0}+2<=T_{1}+0.5$ and $C_{\mathrm{FLO}}: T_{1}+0.5<=$ $T_{0}+2$, respectively.

Since both $C 8 \wedge C_{\mathrm{GO} 2} \wedge T_{2}=T_{0}+2$ and $C 8 \wedge$ $C_{\text {FLO }} \wedge T_{2}=T_{1}+0.5$ are satisfiable, $\left(\left\langle T_{0}\right\rangle\right.$, GasOff 2$)$ and $\left(\left\langle T A, T_{1}, T A\right\rangle\right.$, FlameLightOn $)$ are in fact symbolic enablings in $S 8$. However it is important to note that $C 8 \Rightarrow$ $C_{\mathrm{GO} 2} \wedge T_{2}=T_{0}+2$, i.e., all the markings represented by $S 8$ enable the transition GasOff2. Instead $C 8 \nRightarrow C_{\text {FLO }} \wedge T_{2}=$ $T_{1}+0.5$, i.e., only a subset of the markings expressed by $S 8$ enable the transition FlameLightOn. This is highlighted in the graph by the white tail of the edge from $S 8$ to $S 9$.

\footnotetext{
${ }^{1}$ this picture has been automatically obtained by using GraphViz visualization software [9] on the output generated from the tool-set.

${ }^{2}$ i.e., the set of firing times of a (strong) transition in $S$ also depends on the enablings of the other strong transitions
} 


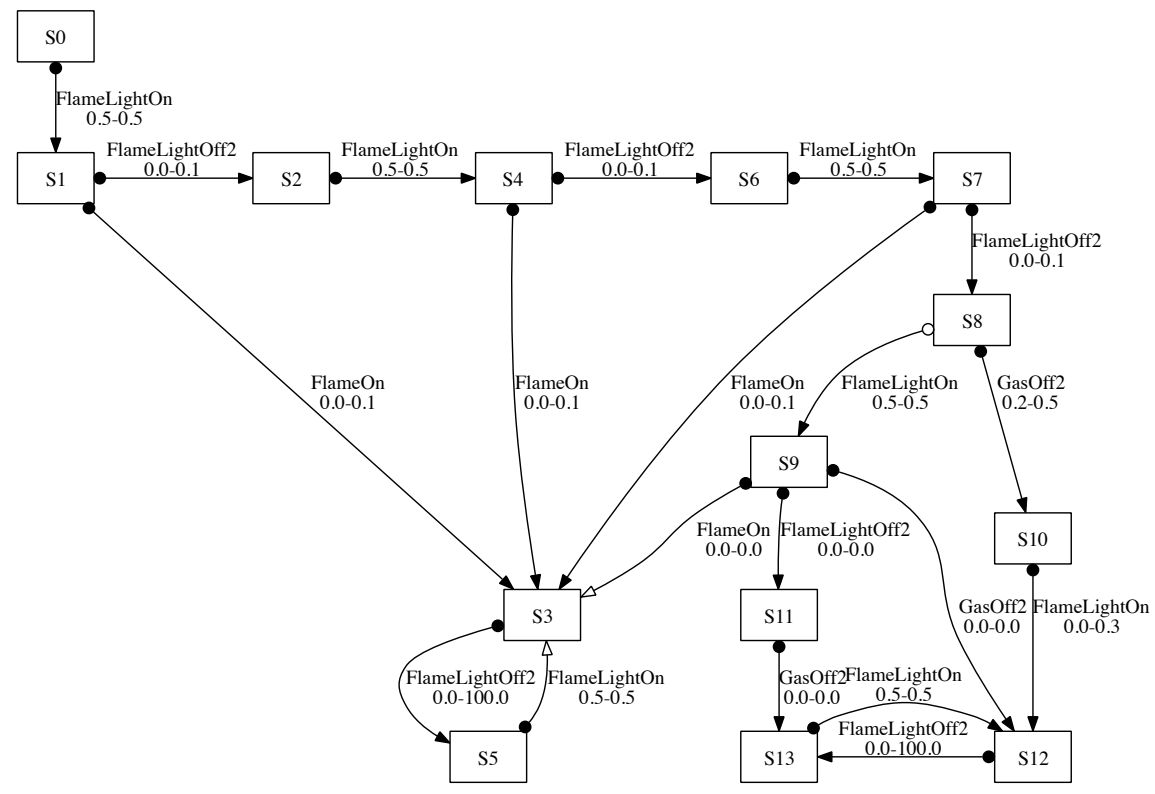

Fig. 2. sample reachability graph

Consider now the firing of $\left(\left\langle T_{0}\right\rangle\right.$, GasOff 2$)$ : it only consumes tokens. In such cases the symbolic firing rule slightly differs from the original one. A second special symbol, $T L$ (Time Last), is introduced. $T L$ can occur only on the constraint of a symbolic state and has an intuitive meaning: it stands for the last firing time of the TB net and it permits a correct interpretation of the model's time semantics. ${ }^{3}$ The reached symbolic state $S 10$ is formally described as:

$$
\begin{aligned}
& M 10: \operatorname{Gas}\left\{T_{1}\right\} \text { Ignition }\left\{T_{A}\right\} \text { NoFlame }\left\{T_{A}\right\} \\
& C 10: \operatorname{Cr} \wedge T_{2}=T_{0}+2 \wedge T L=T_{2}
\end{aligned}
$$

The normalization step eliminates symbols $T_{2}$ (the symbolic firing time) and $T_{0}$, as they occur only in $C 10$, instead it leaves symbol $T L$. That results in (after a timestamp renaming):

$$
\begin{aligned}
& M 10: \operatorname{Gas}\left\{T_{0}\right\} \text { Ignition }\left\{T_{A}\right\} \text { NoFlame }\left\{T_{A}\right\} \\
& C 10: T L \geq T_{0}+0.2 \wedge T L \leq T_{0}+0.5
\end{aligned}
$$

Another circumstance that causes the introduction of $T L$ symbol in a symbolic state representation is when the maximum timestamp symbol $T_{k}$ is replaced with $T A$. How identifying a Time Anonymous in a given symbolic state is the next topic we treat.

The graph in Fig. 2 contains two looping paths: between states $S 3$ and $S 5$, and between $S 12$ and $S 13$ respectively. That happens because in the extrapolated sub-model (Fig. 1), no expected actions are activated after the system exits the ignition phase (e.g., closing the gas valve in the event of

\footnotetext{
${ }^{3}$ in this paper, when $T L$ is left implicit, it is equal to the "last" generated timestamp $T_{k}$.
}

fail, or stopping ignition), so that an unbounded sequence of FlameLightOff2;FlameLightOn is possible.

The white head of the edge from $S 5$ to $S 3$ means that at least one of the ordinary markings represented by $S 3$ is not reachable by following that edge. This happens when a newly built symbolic state is recognized to be strictly included in an existing one. What permits recognizing inclusion between states in this specific case is the usage of Time Anonymous timestamps. $S 3$ is formally defined as:

$$
\begin{aligned}
M 3: & \text { Gas }\{T A\} \text { BURN_PHASE_B }\{T A\} \\
& \text { Ignition }\left\{T_{0}\right\} \text { Flame }\left\{T_{1}\right\} \\
C 3: & T_{1} \geq T_{0} \wedge T_{1} \leq T_{0}+0.1
\end{aligned}
$$

Without using $T A$ s, its original definition $\left(S 3^{\prime}\right)$ would be:

$$
\begin{aligned}
M 3^{\prime}: & \operatorname{Gas}\left\{T_{0}\right\} \text { BURN_PHASE_B }\left\{T_{1}\right\} \\
& \text { Ignition }\left\{T_{0}\right\} \text { Flame }\left\{T_{1}\right\} \\
C 3^{\prime}: & T_{1} \geq T_{0} \wedge T_{1} \leq T_{0}+0.1
\end{aligned}
$$

Let us figure out what would be the model evolution from $S 3^{\prime}$, without introducing $T A$. After the firing sequence FlameLightOff2;FlameLightOr $\sqrt{4}$ a state $S 3^{\prime \prime}$ would be reached, defined in turn as:

$$
\begin{aligned}
M 3^{\prime \prime}: & \quad \text { Gas }\left\{T_{1}\right\} \text { BURN_PHASE_B }\left\{T_{0}\right\} \\
& \text { Ignition }\left\{T_{1}\right\} \text { Flame }\left\{T_{1}\right\} \\
C 3^{\prime \prime}: & T_{1} \geq T_{0}+0.5 \wedge T_{1} \leq T_{0}+100.5
\end{aligned}
$$

Since $S 3^{\prime \prime} \nsubseteq S 3^{\prime}$ and $S 3^{\prime} \nsubseteq S 3^{\prime \prime}$, there is no possibility to merge them and in fact the analysis tool would produce an infinite firing sequence.

\footnotetext{
${ }^{4}$ we omit in this description symbolic enablings, the TB net being safe
} 
Back to $S 3$, we note it corresponds to $S 3^{\prime}$ but for holding $T A$ symbols in places $B U R N \_P H A S E \_B$ and Gas instead of $T_{1}$ and $T_{0}$, respectively. Token $T_{1}$ in $B U R N \_P H A S E \_B$ however is not (and will never be) involved in any symbolic enabling because $B U R N \_P H A S E \_B$ has an empty postset, so it is immediately marked as TA. Token $T_{0}$ in Gas instead is in the preset of transitions FlameLightOn and FlameLightOff2. As for FlameLightOn, the tokens in place Ignition and in place Gas carry on the same timestamp, so either of them is enough to correctly evaluate transition's time function. As for FlameLightOff2, the token in place Gas carries on redundant information due to the simultaneous presence of $T_{1}$ in Flame, that superseded it.

$S 3^{\prime \prime}$ seems really different from $S 3$, but nearly the same heuristics permits us to replace $T_{0}: B U R N_{-} P H A S E \_B$ ( $T_{i}: p$ denotes the occurrence of a timestamp on a place) and $T_{1}$ : Gas with TAs. That eliminates all the occurrences of $T_{0}$ from the marking. After timestamp renaming, we obtain the normal form:

$$
\begin{aligned}
M 3^{\prime \prime}: & \text { Gas }\{T A\} \text { BURN_PHASE_B }\{T A\} \\
& \text { Ignition }\left\{T_{0}\right\} \text { Flame }\left\{T_{0}\right\} \\
C 3^{\prime \prime} \quad: \quad \text { true } &
\end{aligned}
$$

However there is still a difference with respect to $S 3$ : places Ignition and Flame hold the same timestamp, but this boils down to a condition already represented by $S 3\left(T_{1}=T_{0} \Rightarrow\right.$ $C 3$ ), so $S 3^{\prime \prime}$ is recognized as a state included in $S 3$.

Notice that the other cycle on the graph, between $S 12$ and $S 13$, is due to the adoption of a relative notion of time, i.e., it does not depend on the introduced $T A$ concept.

An important setting of the legacy tool [4] was the time limit, a positive interval time that guaranteed the finiteness of the symbolic reachability tree of a TB net. Upon elimination of absolute time references it has been substituted by a relative time limit. This positive interval specifies the maximum admissible distance between different timestamps in a state, and allows one to deal with possibly infinite reachability graph. The tool-set checks whether a symbolic state includes any ordinary states for which the distance between $T L$ and $T_{0}$ (the oldest meaningful timestamp) exceeds the time limit, marking that state as not to be expanded. The rationale behind is that reaching such a user defined limit might be a symptom of the presence of unrecognized "dead tokens", reintroducing absolute time references. If we analyzed the running example disabling $T A$ recognition, the resulting graph would be infinite, unless a time limit is set. Setting this limit to 3 (time units), 25 symbolic states would be generated: 13 already included in the presented graph, the others corresponding to a partial unrolling of the loop between $S 3$ and $S 5$.

The output generated by the tool-set associates a couple of numerical values to edges of the graph, corresponding to the minimum and maximum time distances from the source node to the target node. This permits us to partially recover time relations between nodes that were lost due to the removal of absolute times references from constraints. In the following section we'll show how to exploit them.

\section{Formal Definitions}

Let us formalize some core concepts previously outlined, focusing in particular on $T A$ and coverage.

Definition 1 (well-defined erasure). Let $g_{t}$ be the formal expression of a linear function. The erasure of a set of symbols $E \subset{ }^{\bullet} t$ from $g_{t}$ will be denoted $g_{t[\neg E]} \cdot g_{t[\neg E]}$ is well-defined if it doesn't violate the arity of any operators occurring on $g_{t}$.

Consider for instance $t$, s.t. ${ }^{\bullet} t=\left\{p_{1}, p_{2}\right\}$, and $f_{t}$ : $[\max (\{p 1, p 2\}), p 2+0.5]$, where, $\max : 2^{\mathbb{R}^{+}} \backslash \emptyset \rightarrow \mathbb{R}^{+}$, $+: \mathbb{R}^{+}, \mathbb{R}^{+} \rightarrow \mathbb{R}^{+}$. Then, the erasure $f_{t\left[\neg\left\{p_{1}\right\}\right]}$ is welldefined and results in $[p 2, p 2+0.5]$, instead $f_{t\left[\neg\left\{p_{2}\right\}\right]}$ is not well-defined.

A symbolic instance of $t$ is a mapping $e n_{s}:{ }^{\bullet} t \rightarrow T S \cup$ $\{\mathrm{TA}\}$. Let $e n_{s}^{-1}(\tau)=\{p\}, e n(p)=\tau$.

Let $\mathbf{R}(S)$ be the set of symbolic states reachable from $S$

Definition 2 (valid TA-replacement). Given a state $S, a$ timestamp occurrence $T_{i}: p$ is replaceable with $T A: p$ if and only if for each $S^{\prime}=\left\langle M^{\prime}, C^{\prime}\right\rangle \in \mathbf{R}(S)$ in which token $T_{i}: p$ is left (modulo timestamp renaming), for each symbolic enabling $\left(e n_{s}, t\right)$ in $S^{\prime}$ s.t. $e n_{s}(p)=T_{i}, f_{t[\neg\{p\}]}$ is a welldefined erasure and

$$
\begin{gathered}
C^{\prime} \wedge \max \left(\left\{T L, l b_{t}\left(e n_{s}\right)\right\}\right) \leq u b_{t}\left(e n_{s}\right) \Leftrightarrow \\
C^{\prime} \wedge \max \left(\left\{T L, l b_{t[\neg\{p\}]}\left(e n_{s}\right)\right\}\right) \leq u b_{t[\neg\{p\}]}\left(e n_{s}\right)
\end{gathered}
$$

The semantics of a symbolic state (possibly) including $T A$ is provided by the following coverage notion.

Definition 3 (symbolic state coverage). Let $S=\langle M, C\rangle$ be a symbolic state. An ordinary marking $m$ is covered by $S$ if and only if it corresponds to a numerical substitution $\sigma$ of symbols occurring on $M$, s.t. $\sigma$ satisfies $C$ and for each ordinary enabling $(e n, t)$ in $m$, for each symbolic tuple $\left(e n_{s}, t\right)$ in $S$ s.t. en is a numerical substitution of en ${ }_{s}$,

$$
\begin{aligned}
& \text { a } l b_{t\left[\neg e n_{s}^{-1}(T A)\right]}, u b_{t\left[\neg e n_{s}^{-1}(T A)\right]} \text { are well defined } \\
& \text { b } l b_{t\left[\neg e n_{s}^{-1}(T A)\right]}(e n) \stackrel{=}{=} l b_{t}(e n) \wedge u b_{t_{\left[\neg e n_{s}^{-1}(T A)\right]}(e n)=} \\
& \quad u b_{t}(e n)
\end{aligned}
$$

The next lemma sets the relationship between ordinary and symbolic instances (state transitions).

Lemma 1. Let $m$ be covered by $S$. If $m\left[(e n, t)>m^{\prime}\right.$, then there exists a symbolic enabling ens, s.t. en is a numerical substitution of en $n_{s}, S\left[\left(e n_{s}, t\right)>S^{\prime}\right.$ and $m^{\prime}$ is covered by $S^{\prime}$

Let us finally report as an example some heuristics, including the ones used by the algorithm to identify the $T A$ replacements commented in the previous section. They identify, precisely speaking, a valid replacement of a timestamp occurrence $T_{k}: p$ with $T A: p$, in $S=\langle M, C\rangle$, according to definition 2 .

1) $p^{\bullet}=\emptyset$

2) $\forall t \in p^{\bullet} \bigvee_{i} E_{i}$, where

$E_{1}: f_{t}$ does not refer to $p$, directly or by means of enab

$E_{2}: f_{t}$ is in the form $\left[e n a b+c, e n a b+c^{\prime}\right] \wedge \exists p^{\prime} \in \bullet^{\bullet} t$ $M\left(p^{\prime}\right)=\emptyset \vee\left(\forall T_{j} \in M\left(p^{\prime}\right) C \Rightarrow T_{j} \geq T_{k}\right)$

$E_{3}: \ldots$ 


\section{Property Evaluation}

The symbolic (time coverage) reachability graph contains several exploitable information.

The tool recognizes deadlocks even if they are topologically hidden by the presence of outgoing edges. In fact if all the outgoing edges have a white tail, it is still possible that a proper subset of the corresponding symbolic state is composed by deadlock marking. In the running example however no deadlock marking is reachable.

Disregarding time specification (i.e., considering only the number of tokens distributed over places), the graph nodes exactly identify all the reachable (topological) markings: if a marking matches a symbolic node then there exists at least one path from the initial state to such a marking, conversely if a marking matches no symbolic nodes, it is not reachable. It is thereby possible to verify P-invariants from a specified marking. In case of finite graph, it is possible to answer questions about maximum (minimum) number of tokens in some (combinations) of places.

In general, the set of ordinary (TB net) states represented by the sum of states of the symbolic graph built from a TB net is a superset of the reachable ordinary states of the TB net. In fact, the introduction of TA symbols causes a loss of information, because each TA covers a potential set of timestamps. However, given a symbolic state $S=\langle M, C\rangle$ in which a set $\left\{T_{i}\right\}$ of time-stamp symbols occur on $M$, each numerical substitution of $\left\{T_{i}\right\}$ satisfying $C$ corresponds to the projection of reachable ordinary states. If we are interested in checking timing relations between token's timestamps on the states of the graph we can get three different answers upon graph inspection: a positive one (e.g., there exists a node that satisfies the condition), a negative one (e.g., no nodes satisfy the condition), or a possibly positive. For example, if we are looking for a state where a token in place Flame carries on a timestamp greater than the one in place IGNITION_PHASE_S, state $S 9$ provides us with a positive answer. Instead, if we are checking whether places Gas and Ignition can ever hold the same timestamp the answer is may be (the presence of TA in either places covers that condition).

As for timing relations between token's timestamps in different markings, or between firing times in a transition firing sequence, the symbolic graph permits identifying critical paths by combining the information on edges. In particular, conservative bounds can be established. In the case they are not enough to exclude incorrect timing behaviors, it is possible to carry out a more accurate analysis by rebuilding a portion of the graph, retracing some critical paths and reintroducing absolute time references. For example, looking at the time information on edges, it is possible to establish that state $S 10$ is not reachable from $S 0$ in less than 1.7 time units. We cannot directly infer that $S 10$ is reachable in exactly 1.7 time units.

Concerning feasibility of firing sequences, the symbolic graph expresses all the possibilities (an ordinary firing sequence is matched by any firing sequence on the graph). A critical situation is presented in Fig. 3 If we follow a white- arrow edge (meaning that we reach only a subset of the target state) and, from there, a white-tail edge (meaning that the transition is enabled only in a subset of the ordinary states represented by the node), there is still the possibility that this path actually is not feasible. Also such critical paths could be retraced. Let us stress (back to the reachability problem) that by construction, for every node on the graph there exists a path from the initial state to such a node formed exclusively by black-arrow edges.

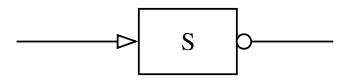

Fig. 3. Critical case for path feasibility

The available tool's evaluation component is still very simple, its integration with some existing model checking engines is currently under investigation. However it already permits examining the input graph looking for interesting properties on topological definition of markings:

- existence of a state with a marking satisfying a constraint (i.e., a boolean combination of condition on the number of tokens in places)

- maximum (minimum) value of an expression involving the number of tokens in places (possibly restricting the evaluation to markings satisfying a given constraint)

\section{TOOL ARChitecture}

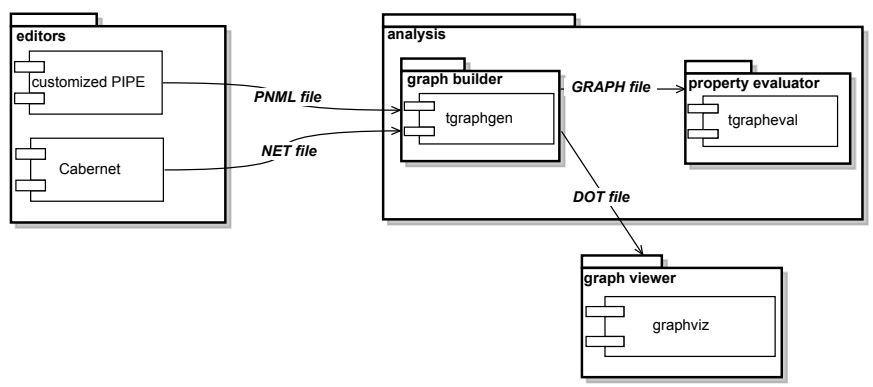

Fig. 4. reference architecture

The analysis technique described in this paper has been implemented as a command line tool written in Java. The tool architecture depicted in Fig. 4 presents the various components that communicate by means of files. The tgraphgen module receives as input a Time Basic Petri net (either in the legacy file format used by the Cabernet tool, or in a PNML format generated, for example, by a customized version of PIPE2 open source tool[10]). It generates as outputs the graph in binary format (used by the property verification module tgrapheval), and in an annotated DOT text format (used by the GraphViz tool). The tool is going to be integrated as an analysis module in the customized PIPE open source tool. That will permit accessing all the functions by means of menu, and exploiting in an integrated environment consolidated structural 


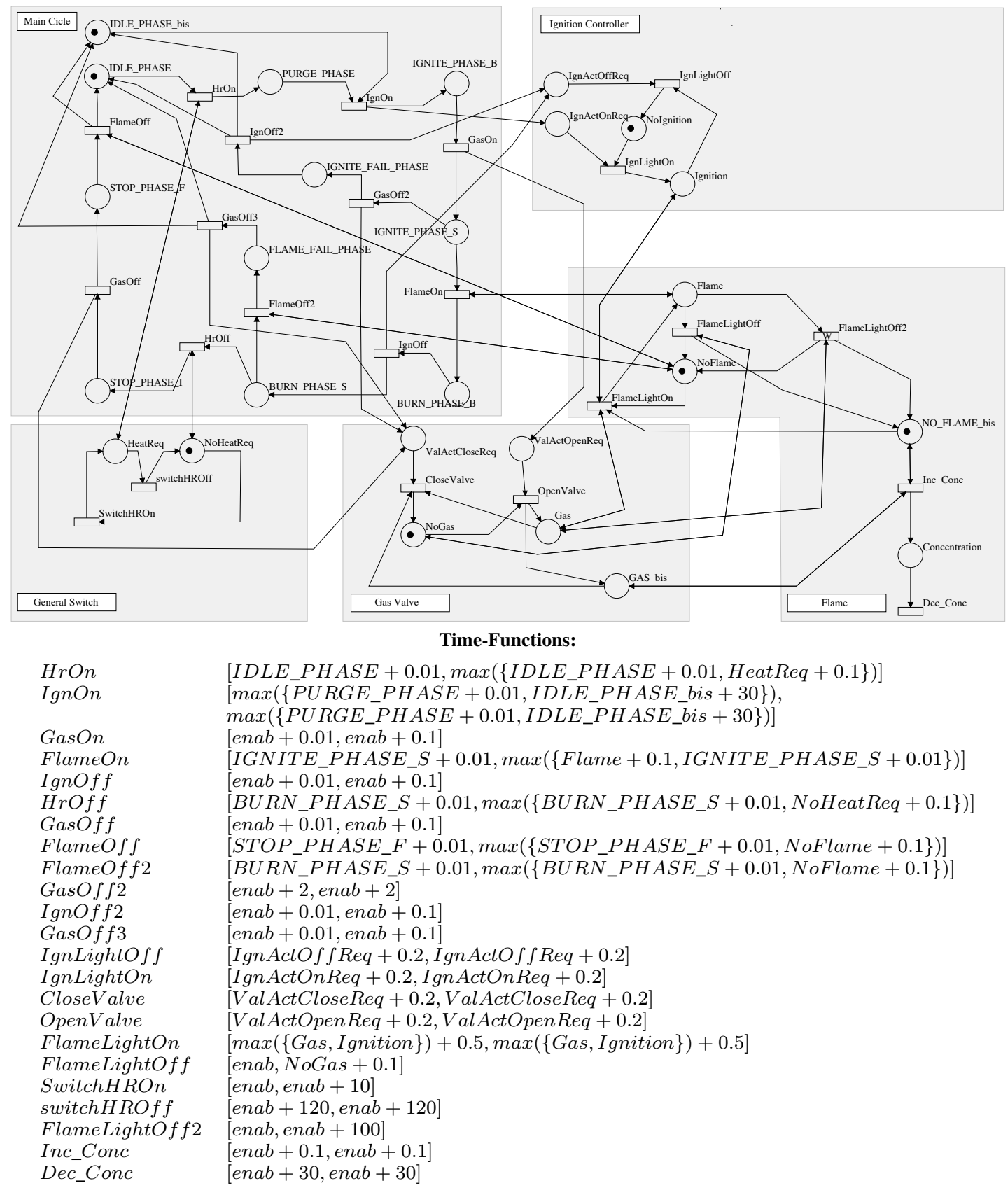

Fig. 5. Use case Net: Gas Burner

analysis algorithms for the verification of the untimed part of TB nets (e.g., P/T nets invariant analysis).

\section{USE CASE AND COMPARISON WITH OTHER TOOLS}

In order to make a comparison with the previous analysis techniques and tools available for TB nets, we consider now the complete gas burner example (Fig. 5), already analyzed in [6].

The main critical parameter of the system was identified in the maximum concentration value of uncombusted gas. With the old analyzers it was only possible to do an approximate analysis, by verifying the safety requirement having fixed a time threshold [6], or by building a small part of the reachability tree able to invalidate the property [11]. A significant improvement of the new tool-set against the old analyzer is that it is now possible to compute the upper bound for such a parameter.

Table I reports the outcomes of the analysis on the use case. In particular the considered parameter has been measured with three versions of the net. They differ in the time granularity used for the uncombusted gas process, i.e., the time function of the transition Inc_Conc. The first thing to note is however 
that the analysis result is coherent in the various situations, identifying the maximum amount of uncombusted gas as corresponding to a leaking period of two seconds.

The test has been performed on a Toshiba Notebook with 2.4Ghz Intel Core 2 Duo processor and 4GB of memory. The operating system is Ubuntu 10.10 and the Java Virtual Machine is OpenJDK IcedTea6 1.9.5.

On the table we report also the number of states of the final reduced graph against the overall number of states generated by the algorithm, and the execution times. Even if the sample is too little, a first interpolation suggests that a likely trend is quadratic (with a small constant factor).

In Fig. 6 some profiling data -relating the 0.1 time granularity version of the model- are presented. On the $\mathrm{x}$ axis there is the execution time expressed in minutes, on the $y$ axis there are the number of built nodes, of reduced (final) nodes, and of nodes ready to be processed, respectively. This picture is important for two reasons: first it shows that the performance degradation of state construction process is very small (the number of states created is pretty much constant in time after an initial burst); second, it supports the idea that a parallel (distributed) version of the graph builder, currently under development, should substantially improve the performances (the front of expansion remaining consistently wide).

TABLE I

Use CASE ANALYsis Results

\begin{tabular}{|c|c|c|c|}
\hline \hline Inc_Conc granularity & $\max ($ Conc) & \# [final/built] states & exec. time \\
\hline \hline 0.5 & 4 & $865 / 1217$ & $\approx 75$ secs \\
\hline 0.25 & 8 & $2233 / 2983$ & $\approx 400$ secs \\
\hline 0.1 & 20 & $14563 / 23635$ & $\approx 7.5 \mathrm{hrs}$ \\
\hline
\end{tabular}

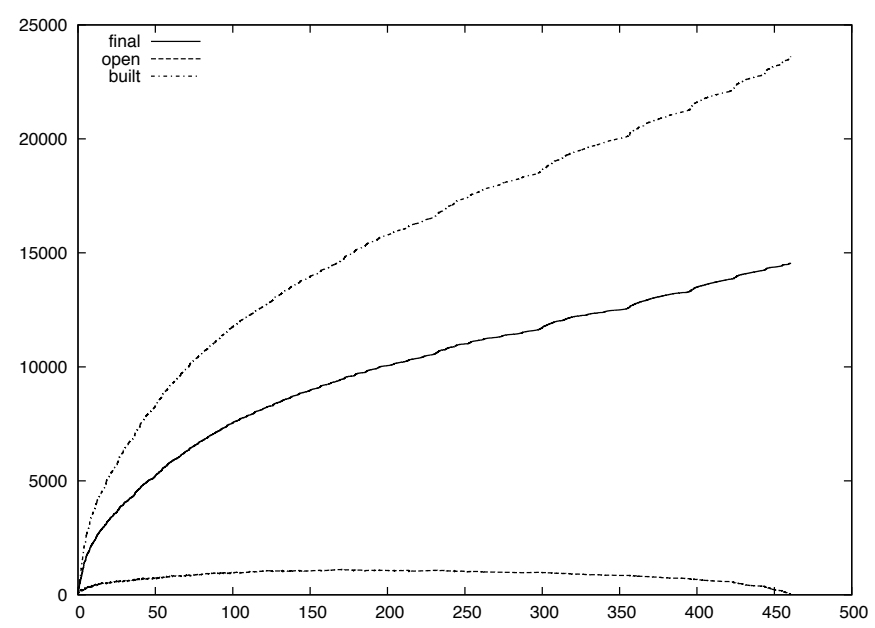

Fig. 6. state creation advancement

\section{CONCLUSION AND FUTURE WORKS}

The analysis technique presented in this paper overtakes the existing available analysis technique for Time Basic Nets (a very expressive timed version of Petri nets) because it permits the building of a sort of (symbolic) time-coverage reachability graph keeping interesting timing properties of the nets. In particular the introduction of the concept of time anonymous timestamps, allows for a major factorization of symbolic states. An extension of the technique that further exploits the time anonymous concept in order to deal with topologically unbounded nets (by means of a coverage of $T A$ tokens, i.e., a sort of $\omega_{\mathrm{TA}}$ ) is under definition.

\section{REFERENCES}

[1] C. Ghezzi, D. Mandrioli, S. Morasca, and M. Pezzè, "A unified high-level petri net formalism for time-critical systems," IEEE Trans. Softw. Eng., vol. 17, pp. 160-172, February 1991. [Online]. Available: http://portal.acm.org/citation.cfm?id=104878.104897

[2] B. Berthomieu and M. Diaz, "Modeling and verification of time dependent systems using time petri nets," IEEE Trans. Softw. Eng., vol. 17, pp. 259-273, March 1991. [Online]. Available: http://dx.doi.org/10.1109/32.75415

[3] A. Kovacs and S. Hudak, "Time semantics in time basic nets," in Applied Machine Intelligence and Informatics (SAMI), 2010 IEEE 8th International Symposium on, 2010, pp. 315 -319.

[4] C. Ghezzi and M. Pezzè, "Towards extensible graphical formalisms," in Proceedings of the 7th international workshop on Software specification and design, ser. IWSSD '93. Los Alamitos, CA, USA: IEEE Computer Society Press, 1993, pp. 69-77. [Online]. Available: http://portal.acm.org/citation.cfm?id=951807.951821

[5] A. P. Atlee and H. Gannon, "Specifying and verifying requirements of real-time systems," IEEE Trans. Softw. Eng., vol. 19, pp. 41-55, January 1993. [Online]. Available: http://portal.acm.org/citation.cfm? id=630793.631022

[6] C. Bellettini, M. Felder, and M. Pezzè, "A tool for analysing highlevel timed petri nets," Politecnico di Milano, IPTES Esprit Project 5570 PDM-41, Sep. 1993.

[7] C. Bellettini, M. Felder, and M. Pezzè, "Merlot: a tool for analysis of real-time specifications," in Proceedings of the 7th international workshop on Software specification and design, ser. IWSSD '93. Los Alamitos, CA, USA: IEEE Computer Society Press, 1993, pp. 110-119. [Online]. Available: http://portal.acm.org/citation.cfm?id= 951807.951826

[8] C. Ghezzi, S. Morasca, and M. Pezzè, "Validating timing requirements for time basic net specifications," J. Syst. Softw., vol. 27, pp. 97-117, November 1994. [Online]. Available: http://portal.acm.org/citation.cfm? id=196668.196672

[9] http://www.graphviz.org/, "Graphviz - graph visualization software."

[10] http://pipe2.sourceforge.net/, "Platform independent petri net editor 2."

[11] F. Calzolari and M. Pezzè, "Property decomposition to speed up analysis," Real-Time Systems, Euromicro Conference on, vol. 0, p. 147, 1995. 